\title{
A Theoretical Calculation Method of the Unsupported Span for the Shallow Tunnel in the Soft Stratum
}

\author{
Xiaoxu Tian, ${ }^{1,2}$ Zhanping Song $\mathbb{B D}^{1,2}$ Guannan Zhou, ${ }^{2,3}$ and Xiaowei Zhang ${ }^{4}$ \\ ${ }^{1}$ School of Civil Engineering, Xi'an University of Architecture and Technology, Xi'an, Shaanxi 710055, China \\ ${ }^{2}$ Shaanxi Key Laboratory of Geotechnical and Underground Space Engineering, Xi'an 710055, China \\ ${ }^{3}$ China Railway Bridge Engineering Bureau Group Co., Ltd., Tianjin, 300300, China \\ ${ }^{4}$ CCCC Fourth Highway Engineering Bureau Co., Ltd., Beijing, China \\ Correspondence should be addressed to Zhanping Song; songzhpyt@xauat.edu.cn
}

Received 19 November 2019; Revised 14 July 2020; Accepted 28 July 2020; Published 14 August 2020

Academic Editor: Hui Yao

Copyright $\odot 2020$ Xiaoxu Tian et al. This is an open access article distributed under the Creative Commons Attribution License, which permits unrestricted use, distribution, and reproduction in any medium, provided the original work is properly cited.

\begin{abstract}
During the construction of the tunnel in soft stratum, it is often found that the unsupported span is too large, resulting in instability of the tunnel face and collapse of the vault. However, the unsupported span was often selected according to the experience of engineers in the actual construction process, which was lack of the theoretical basis. Therefore, based on the calculation model of the surrounding rock pressure of shallow buried tunnel, this paper analyzed the stability of the tunnel face and the vault and then obtained the calculation formula of the unsupported span of the shallow buried tunnel in soft rock stratum. It was pointed out that the unsupported span is not determined by the arch crown stability or the tunnel face stability alone, but by both. The rationality of the formula was verified by a centrifugal test and an engineering case. The analysis and discussion showed that the unsupported span is sensitive to the cohesion and internal friction angle of the rock-soil mass, especially the cohesion. The unsupported span of the shallow buried tunnel in the soft rock stratum is a linear function of the support pressure. The support pressure has a more significant contribution to the increase of the unsupported span by the centre cross diaphragm (CRD) method, and the unsupported span increases linearly with the increase of the support pressure. The research results provide a theoretical reference for the determination of the unsupported span for the shallow tunnel in the soft stratum.
\end{abstract}

\section{Introduction}

Recently, with the rapid development of the traffic system, the scale and number of urban railways and mountain tunnels have significantly increased, which means that tunnels in soft strata are also increasing [1-6]. In order to build a tunnel in soft stratum, it is necessary to focus on solving the problems of stability of the vault, stability of the tunnel face, and excessive surface settlement [7-11]. Apart from the surrounding rock classification, tunnel depth, excavation method, and construction method, the unsupported span is also an essential factor affecting the three issues above. For a specific project, surrounding rock classification, tunnel depth, excavation method, and construction method are determined, so the unsupported span is particularly important [12-14]. Especially in construction of tunnels in shallow buried soft stratum, oversizing the unsupported span and reduced stability of soft stratum may cause tunnel face instability and excessive ground surface settlement, which may further cause the arch crown collapse. However, undersizing the unsupported span may significantly increase the cost of tunnel construction. Therefore, it is of great practical significance for the tunnel's safety and cost to study the appropriate unsupported span.

Many scholars explored the determination of tunnel with the unsupported span. In theory, based on the Terzaghi loose rock theory and the Mohr-Coulomb strength criterion, Wang and Gong [15] derived the calculation formula of the unsupported span of the shallow buried underground tunnel and gave the suggestions of increasing the unsupported span and speeding up the construction speed. Based on the Janssen silos theory, Shi et al. [16] proposed the 
calculation formula for the unsupported span of the shallow buried section of the rock mass tunnel entrance, analyzing the reasonable unsupported span under the condition of different mechanical parameters. It was found that the core soil can significantly improve the stability of the tunnel face. The excavation footage is sensitive to the cohesion of surrounding rock, which is unfavourable to the stability of the rock pile tunnel. Zhang et al. [17] established an improved prism-wedge model to reasonably evaluate the safety factor of a tunnel face and an unsupported span in cohesivefrictional soils. In light of Janssen's silo theory and Mohr-Coulomb strength criterion, Liang et al. [18] established a calculation formula of vertical force loading on vaults, based on the basis of logarithmic spiral failure mode, setting up the formula to calculate the round length in advanced core soil on the tunnel face, which is governed by such relevant parameters as internal friction angle, cohesive force, support pressure, and excavation height. In the model experimental research, Lee and Schuber [19] designed the unsupported span model experiment in the sand and used the statistical results of PFC3D numerical software analysis and the relative shear stress to derive the calculation method of the unsupported span of the soft rock tunnel. Although the above studies include calculation formulas for deriving the unsupported span for soil and soft rock tunnels, they are based on the premise of a stable bearing arch, the arch crown, or the face, without considering the stability of the arch crown and the face at the same time. Under different geological conditions or different excavation conditions, oversizing the unsupported span may result in instability of the face and the collapse of the arch crown. Because of the complex mechanical properties of soft rock formations, the stability of both the arch crown and the face should be considered when calculating the unsupported span. For the soft rock stratum, there is much research on the effect of pilesoil arching, but there is little research on the effect of the soil arching covering the top of the tunnel. Therefore, the specific shape and distribution area of the soil arching are still unclear. There is a lack of theoretical basis for the calculation formula of the unsupported span based on the bearing arch. Janssen silos theory is usually used to evaluate the arch effect at the top of the tunnel [20]. According to Horn's experimental study in 1961, Janssen silos theory can also be used to explain the instability mechanism of the face. However, according to the research of many experts in recent years, the actual failure form of the excavation surface is a chimney, which is quite different from the model assumed in the theoretical analysis of the Janssen silos theory [21-24].

Based on the actual failure form of the excavation face, this paper expanded the two-dimensional calculation model of the surrounding rock pressure of the shallow tunnel into a three-dimensional model. Through the analysis of the stability of the tunnel face and the stability of the vault, the calculation formula of shallow tunnel unsupported span was derived. The rationality of the formula was verified by a centrifugal test and an engineering case. The parameters were discussed and analyzed. The research methods and results of this paper provide a reference for the theoretical calculation of the unsupported span for the shallow tunnel in the soft stratum.

\section{A Calculation Method of the Unsupported Span}

At present, there are many theoretical studies on the failure modes of the arch crown and the tunnel face. For example, Janssen silos theory, rock failure mode, parabolic failure mode $[25,26]$, and logarithmic spiral mode [27]. However, in actual engineering, the failure mode of the excavation face is far from the analysis model above. In order to find out the true failure mode of the excavation face, Mair used the centrifuge model test to prove that the failure surface of the cohesive soil layer is extended upward and towards two sides, and it is like a basin, narrow in the lower part and wide in the upper part [28]. Selby also has shown that the failure surface of the sandy soil layer is a narrow chimney shape that directly develops to the surface of the tunnel from both sides [29]. The actual failure mode of the excavation surface is consistent with the test results of Mair. In fact, for shallow tunnels in soft rock stratum, the effects of excavation will extend to the surface, failing to form a "natural arch." After the tunnel is excavated, the rock and soil may collapse and move, which extends to the surface and forms a collapsed area. The failure pattern is shown in Figure 1.

Xie simplified the failure mode and proposed a calculation model of the surrounding rock pressure of the shallow buried tunnel [30] (Figure 2). He also derived the calculation formula of the surrounding rock pressure. After a large number of engineering practices, Xie's surrounding rock pressure calculation method has good applicability in soft rock formations. Therefore, this method had been incorporated into China's Code for Design of Railway Tunnels and is used as a standard for promotion. According to Xie's simplified failure model, this paper derived the formula for calculating the unsupported span of the tunnel in soft rock.

\subsection{A Calculation Model}

(1) The calculation assumes that (1)the rock-soil mass obeys the Mohr-Coulomb criterion and (2)the percolation effect is not considered

(2) Failure mode is presented

Xie's simplified model for calculating the surrounding rock pressure is two-dimensional. The unit-body with a unit length of 1 is taken in the longitudinal direction of the tunnel, and the fracture surface of the rock-soil mass is assumed to extend upward from the corners of the sidewalls of the tunnel. The angle between the fracture surface and the horizontal plane is $\beta$. According to the literature $[28,29]$ and engineering practices, similar fracture surfaces are formed in the face of the tunnel and the unsupported arch rock mass. Thus, it is also assumed that the angle between the fracture face extended from the face and the arch crown and the horizontal plane is $\beta$, as shown in Figure 3(a).

Based on the above assumptions and analysis, this paper proposed a calculation model for the unsupported span of 


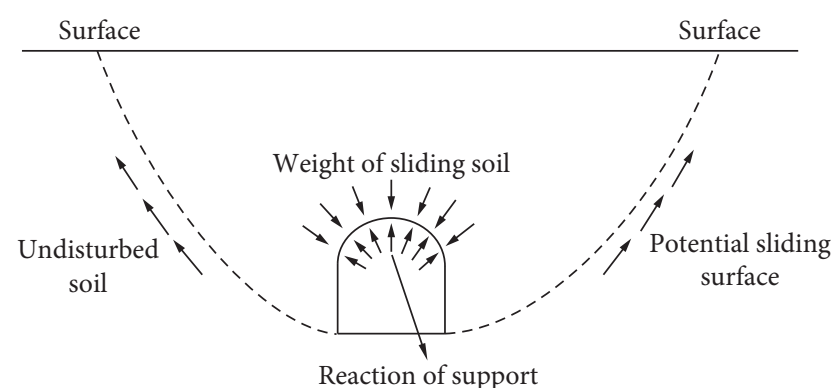

Figure 1: Potential failure shape of the tunnel face.

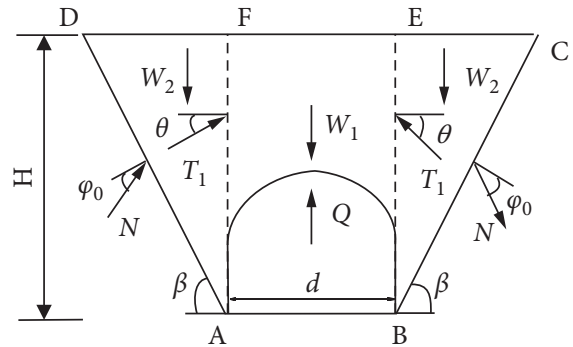

FIGURE 2: Model of surrounding rock pressure of shallow buried tunnel.

tunnels in soft rock stratum. (1) The fracture surface formed in the rock mass is oblique straight, which forms an angle $\beta$ with the horizontal plane. (2) When the rock mass ABEF $\left(\mathrm{AB}^{\prime} \mathrm{E}^{\prime} \mathrm{F}\right)$ covering on the top of the tunnel sinks, it is clamped by the rock-soil mass on both sides, which in turn drives the decline of trigones $\mathrm{AFD}$ and $\mathrm{BCE}\left(\mathrm{B}^{\prime} \mathrm{C}^{\prime} \mathrm{E}^{\prime}\right)$ on both sides. When the entire sliding mass ABCEFD $\left(A B^{\prime} C^{\prime} E^{\prime} F D\right)$ slides, it is subjected to the resistance of the undisturbed rock mass on both sides. And when the $\mathrm{AB}^{\prime} \mathrm{C}^{\prime} \mathrm{E}^{\prime} \mathrm{FD}$ sliding mass slides, it is still subjected to the resistance of the rock-soil mass $\mathrm{OB}^{\prime} \mathrm{B}$ in front of the tunnel face. This is shown in Figures 3(b) and 3(c).

The unsupported span is $s$. According to the unfavourable situation, the initial support lags $s$ after the excavation of the original face. After excavation, the soil mass above the tunnel is loosened. The height of the loose rock mass above the tunnel is $\mathrm{H}$. The length of the loose rock-soil mass above the tunnel is $l=s+b$, where $b$ is the length of the rock-soil mass in front of the face when excavating, and $b=(h / \tan \beta)$. The unsupported span $s$ is controlled by two conditions: (1) after excavation, if the surrounding rock at the top of the tunnel within the loose range does not collapse, the weight of the surrounding rock should be less than the friction resistance, as shown in Figure 3(b); (2) the tunnel face will not collapse, as shown in Figure 3(c).

2.2. Stability Analysis of the Arch Crown. After excavation, to make sure the surrounding rock at the top of the tunnel does not collapse in the loose range, the self-weight of the surrounding rock should be less than the four-side frictional resistance. The equilibrium equation was established by taking the limit. The calculation diagram (Figure 4) is as follows:

$$
W_{1}-2 T_{1} \sin (\theta)-2 T_{2} \sin (\theta)=0,
$$

where $W_{1}$ is the self-weight of rock mass ABEF covering on the top of the cave, 1

$$
W_{1}=s d H \gamma,
$$

and $2 T_{1} \sin (\theta)$ is the friction from the soil on two sides when the rock mass ABEF slides down, and $2 T_{2} \sin (\theta)$ is the friction of the other two sides. $T_{1}$ and $T_{2}$ can be solved as follows; the calculation diagram is shown in Figure 5.

The self-weight of the triangular prism is

$$
\frac{1}{2} \gamma \times \overline{\mathrm{AF}} \times \overline{\mathrm{DF}} \times s=\frac{1}{2} \gamma H^{2} \frac{1}{\tan (\beta)} d .
$$

According to the law of sines, we can get

$$
\frac{T_{1}}{\sin \left(\beta-\varphi_{0}\right)}=\frac{W_{2}}{\sin \left[90^{\circ}-\left(\beta-\varphi_{0}+\theta\right)\right]} .
$$

Put equation (3) into the above formula and get it after simplification:

$$
T_{1}=\frac{1}{2} \gamma H^{2} \frac{\lambda}{\cos (\theta)} d
$$

The same is available:

$$
T_{2}=\frac{1}{2} \gamma H^{2} \frac{\lambda}{\cos (\theta)} s .
$$

In the formula,

$$
\begin{gathered}
\tan (\beta)=\tan \left(\varphi_{0}\right)+\sqrt{\frac{\left(\tan ^{2}\left(\varphi_{0}\right)+1\right) \tan \left(\varphi_{0}\right)}{\tan \left(\varphi_{0}\right)-\tan (\theta)}}, \\
\lambda=\frac{\tan (\beta)-\tan \left(\varphi_{0}\right)}{\tan (\beta)\left[1+\tan (\beta)\left(\tan \left(\varphi_{0}\right)-\tan (\theta)\right)+\tan \left(\varphi_{0}\right) \tan (\theta)\right]} .
\end{gathered}
$$

Put (2), (4), and (5) into (1); the following formula can be obtained:

$$
s=\frac{d H \lambda \tan \theta}{d-H \lambda \tan \theta},
$$

where $s$ is the unsupported span, $d$ is the tunnel width or diameter, $H$ is the thickness of the covered soil layer, and $\phi_{0}$ is the frictional angle. The value of $\theta$ and $\phi_{0}$ is shown in Table 1.

2.3. Stability Analysis of the Face. When the face is in the limit equilibrium state, the calculation diagram (Figure 6) is as follows.

$$
Q=W_{1}^{\prime}-2 T_{1}^{\prime} \sin (\theta)-2 T_{2}^{\prime} \sin (\theta) .
$$

The limit equilibrium equation is established along the tangential direction and the normal direction of the fracture surface $\mathrm{OB}^{\prime}$ : 


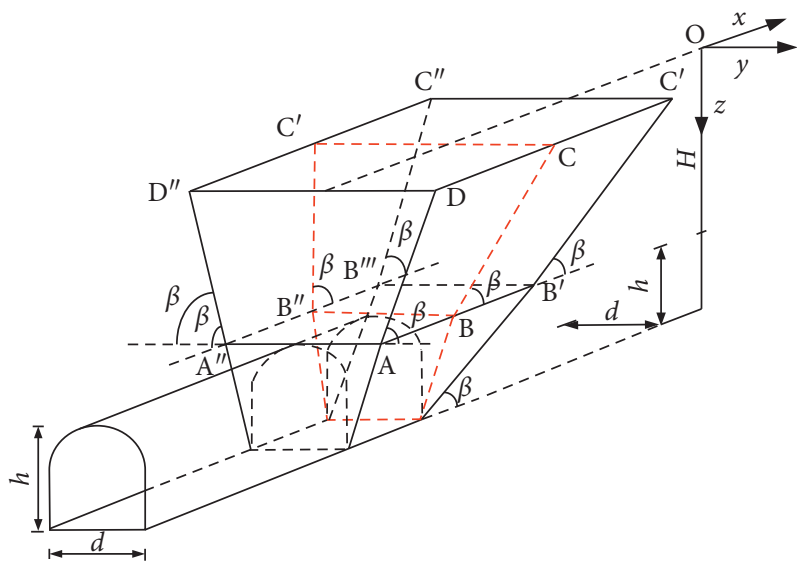

(a)

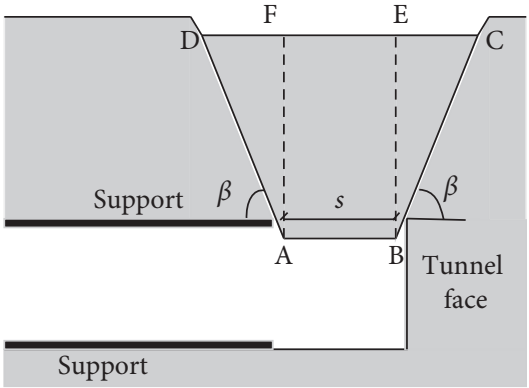

(b)

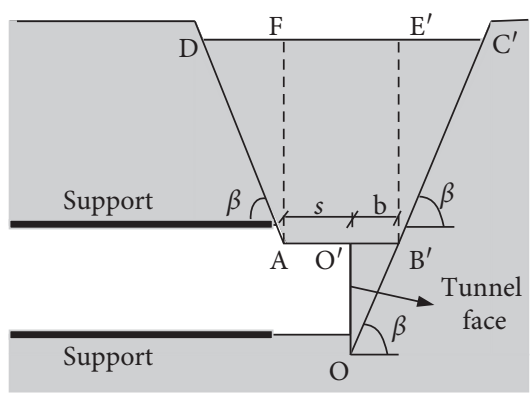

(c)

Figure 3: A calculation model of the unsupported span. (a) Three-dimensional model. (b) Failure mode of the arch crown. (c) Failure mode of the tunnel face.

$$
\left\{\begin{array}{l}
T+T_{n}-\left(Q+W_{3}\right) \sin (\beta)+P \cos (\beta)=0, \\
N-\left(Q+W_{3}\right) \cos (\beta)-P \sin (\beta)=0 .
\end{array}\right.
$$

According to the Mohr-Coulomb criterion,

$$
T=N \tan \left(\varphi_{0}\right)+c d \frac{h}{\sin (\beta)},
$$

where $T_{n}$ is the shear force imposed on the side of the wedge $\mathrm{OO}^{\prime} \mathrm{B}^{\prime}$, and its solution and the calculation diagram (Figure 7) are as follows.

According to the Mohr-Coulomb criterion, the shear force at any point on the sliding surface on either side of the wedge is

$$
\tau(y, z)=c+\sigma_{x}(y, z) \tan \left(\varphi_{0}\right) .
$$

$\sigma_{x}(y, z)$ is the sliding surface normal stress. Assume the relationship between $\sigma_{x}(y, z)$ and the vertical stress $\sigma_{z}(y, z)$ is as follows:

$$
\sigma_{x}(y, z)=K_{a} \sigma_{z}(y, z)
$$

Concerning the influence of tunnel excavation unloading, $K_{a}$ is the active earth pressure coefficient, and it is assumed that the vertical stress of the wedge is linearly distributed along with the depth. Then the vertical stress is

$$
\sigma_{z}(y, z)=(h-z) \gamma+\frac{z}{h} \sigma_{v}
$$

where $\sigma_{v}=(Q / b d)$ and $h$ is the height of the tunnel section.

From (13)-(15), the following formula can be obtained:

$$
\tau(y, z)=c+K_{a} \tan \left(\varphi_{0}\right)\left[(h-z) \gamma+\frac{z}{h} \sigma_{v}\right] .
$$

Integrate (16) along with the height of the wedge:

$$
T_{n}=2 \int_{0}^{h} \tau(y, z) b(z) d z
$$

$b(z)$ is the width of the corresponding wedge in the height of $z$ :

$$
b(z)=\frac{z}{\tan (\beta)} .
$$

Put (18) into (17); the following formula can be obtained:

$$
T_{n}=\frac{h^{2}}{\tan \beta}\left(c+K_{a} \tan \varphi_{0} \frac{2 \sigma_{v}+\gamma h}{3}\right) .
$$

Put (12) into (11) and eliminate $N$; the following formula can be obtained:

$$
\begin{aligned}
& c d \frac{h}{\sin (\beta)}+T_{n}-\left(Q+W_{3}\right) \sin (\beta)+P \cos (\beta) \\
& \quad+\left(Q+W_{3}\right) \cos (\beta) \tan \left(\varphi_{0}\right)+P \sin (\beta) \tan \left(\varphi_{0}\right)=0 .
\end{aligned}
$$




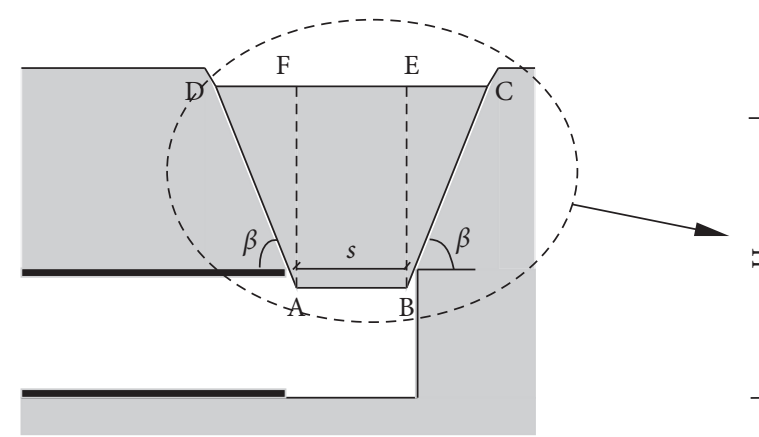

(a)

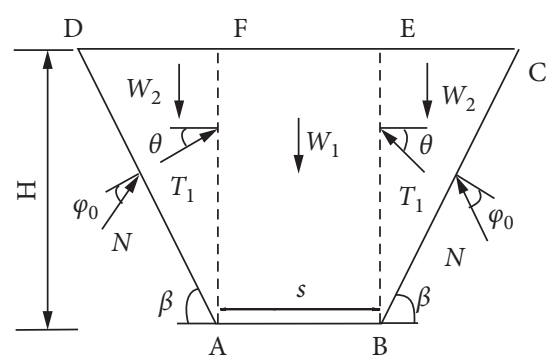

(b)

Figure 4: Calculation diagram for stability analysis of the arch crown. (a) Failure mode. (b) Calculation diagram.
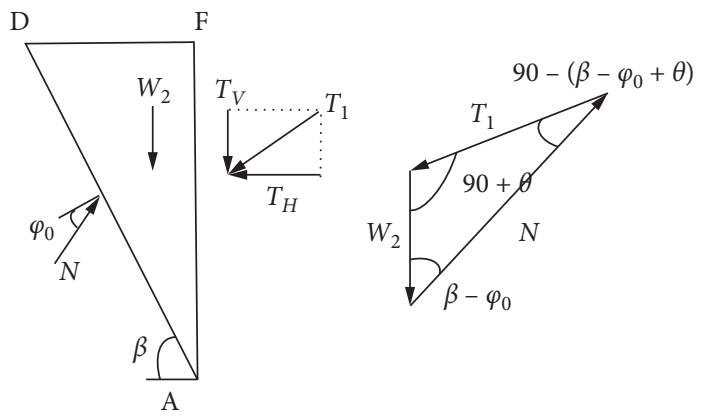

FIgURE 5: Calculation diagram.

Table 1: $\theta$ and $\phi_{0}$ value of all grade of surrounding rock (China's Code for Design of Railway Tunnels).

\begin{tabular}{lcccccc}
\hline Grade & I & II & III & IV & V \\
\hline$\theta\left({ }^{\circ}\right)$ & $0.9 \phi_{0}$ & $0.9 \phi_{0}$ & $0.9 \phi_{0}$ & $(0.7-0.9) \phi_{0}$ & $(0.5-0.7) \phi_{0}$ & VI \\
$\phi_{0}\left({ }^{\circ}\right)$ & $>78$ & $70 \sim 78$ & $60 \sim 70$ & $50 \sim 60$ & $40 \sim 50 \quad 0.3-0.5) \phi_{0}$ \\
\hline
\end{tabular}

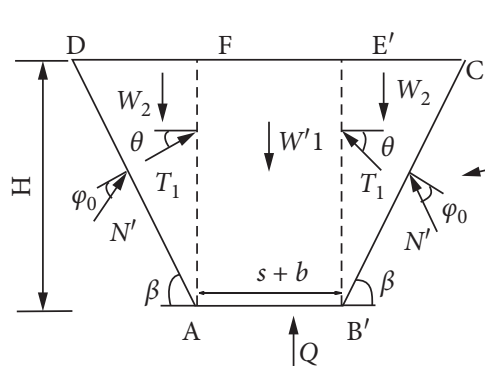

(a)

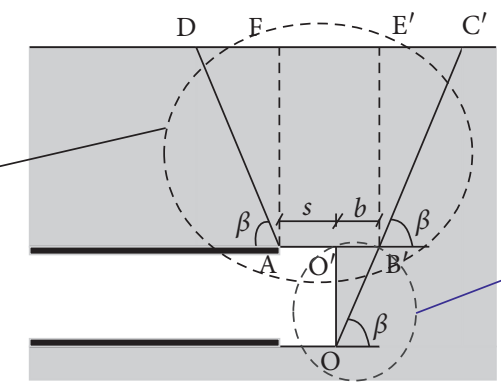

(b)

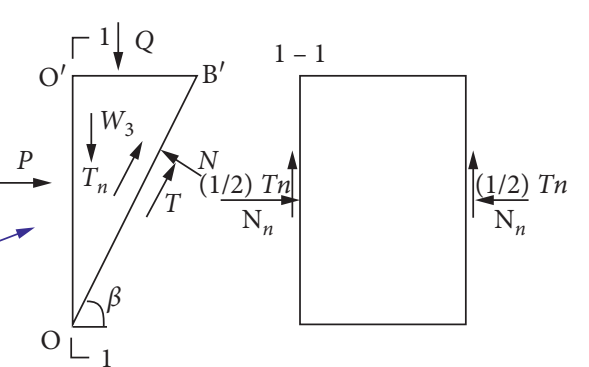

(c)

FIgURE 6: Calculation diagram for stability analysis of the face. (a) Calculation diagram. (b) Failure mode. (c) Calculation diagram.

Then put (10) and (19) into (20), the following formula can be obtained:

$$
\begin{aligned}
s= & \frac{k_{3}}{\left(d H \gamma-\gamma H^{2} \lambda \tan (\theta)\right)\left(k_{2}-\left(2 h^{2} / 3 b d\right) K_{a} k_{1}\right)} p \\
& +\frac{(b+d) H \lambda \tan (\theta)-b d}{d-H \lambda \tan (\theta)}+\frac{c h((d / \sin (\beta))+(h / \tan (\beta)))+(1 / 3) \gamma h^{3} K_{a} k_{1}-k_{2} W_{3}}{\left(d H \gamma-\gamma H^{2} \lambda \tan (\theta)\right)\left(k_{2}-\left(2 h^{2} / 3 b d\right) K_{a} k_{1}\right)},
\end{aligned}
$$




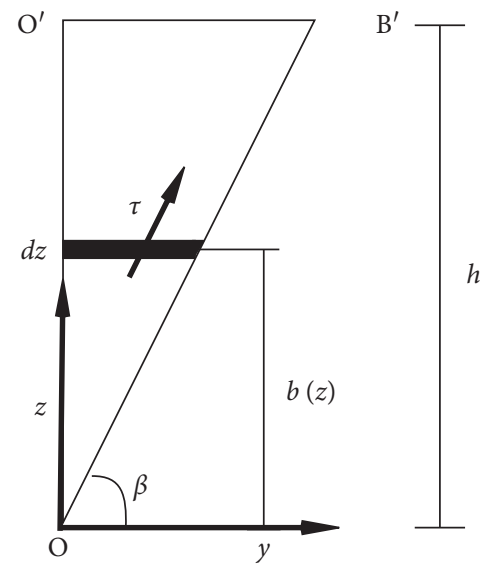

Figure 7: Calculation diagram.

where $k_{1}=\left(\tan \left(\phi_{0}\right) / \tan (\beta)\right), k_{2}=\sin (\beta)-\cos (\beta) \tan \left(\phi_{0}\right)$, $k_{3}=\cos (\beta)+\sin (\beta) \tan \left(\phi_{0}\right)$, and $p$ is the joint force of the support pressure of the face, the spray effect of the face, and the support of the core soil to the face.

The formulas derived in 2.2 and 2.3 above apply to shallow buried tunnels. The deep buried depth $H$ of the tunnel should meet $h_{a}<H \leq(2-2.5) h_{a}$, and $h_{a}$ is the equivalent load height. Unsupported span $s$ should ensure that the face and the arch crown are stable; that is, the minimum values of (9) and (21) are obtained: $s=\min \{(6),(18)\}$.

\section{Cases Study}

3.1. The Model Test. The model test is from the literature [19]. The scale for the models is 1:40 and tunnel lining is modelled by a plastic plate and ground is modelled by sand classified as SW (well-graded sand) according to the Unified Soil Classification System. The chosen water content was 1.6-2.0\%, which results in an apparent cohesion. Its friction angle is approximately $30^{\circ}-32^{\circ}$, and apparent cohesion is approximately $0.5-1.5 \mathrm{kPa}$, measured by the indirect method. Tunnel diameter is $25 \mathrm{~cm}(10 \mathrm{~m})$, and overburden is $50 \mathrm{~cm}(20 \mathrm{~m})$, and half-space is modelled. The excavation was carried out in $1.25-2.5 \mathrm{~cm}(0.5-1.0 \mathrm{~m})$ interval to investigate the influence of the round length on the stability of the face and unsupported span. The unsupported span of the face stability was calculated to be $1.1 \mathrm{~m}$, and the unsupported span of the arch crown stability is $3.7 \mathrm{~m}$ according to the formulas derived in the paper. The model test results were shown in Table 2 and Figure 8. When the unsupported span exceeded $1.1 \mathrm{~m}$, the tunnel face began to collapse. When the unsupported span exceeded $3.7 \mathrm{~m}$, both the tunnel face and the arch crown began to collapse. The calculation results are consistent with the experimental results. It can be seen that the formula derived in this paper is rational.

3.2. Practical Engineering. Tianheng Mountain Tunnel is located in Tuanjie Town, Harbin City, and is the critical project of the northeast section of Harbin Ring Expressway. The tunnel adopts a two-line split type with an uplink length of $1,660 \mathrm{~m}$ and a downlink length of $1,690 \mathrm{~m}$. The tunnel crosses Tianheng Mountain. Most of the surrounding rocks are cohesive soils with local sand layers, and surrounding rock type is V VI. The tunnel was constructed by the New Austrian Tunnelling Method and has a buried depth of $28 \sim 40 \mathrm{~m}$. It is a typical shallow buried earth tunnel. The section height is $10 \sim 13 \mathrm{~m}$, and the diameter is $12 \sim 16 \mathrm{~m}$. When the section area is $126 \mathrm{~m}^{2}$, the bench cut method was used to construct it. When the section area is $163 \mathrm{~m}^{2}$, the bench cut method or CD and CRD methods are used. The calculation parameters are shown in Table 3.

The formula provided by this paper and other scholars was adopted to calculate the unsupported span, as shown in Table 4. The calculation results of the unsupported span of this paper are less than the literature $[15,16]$. This indicated that the calculation results of the unsupported span in this paper could meet the requirements of the literature [15] and the literature [16]. When using the CRD method, the literature [15] obtained $d+14.2 \geq 0$, indicating that the unsupported span has not been controlled by the stability of the arch crown, and the unsupported span can be arbitrarily selected. It means that when the calculation formula of the unsupported span is established by arch crown stability alone, there may be no result. The formula provided by the literature [16] cannot directly calculate the unsupported span. Only a series of excavation dimensions can be drawn up in advance, and then it is verified whether the face is stable and finally the optimal size is selected. Obviously, the unsupported span calculated in this paper with CRD method is $4.8 \mathrm{~m}$, and the bench cut method is $1.2 \mathrm{~m}$, which is consistent with the actual value used on site.

When changing from the bench cut method to the CRD method, the unsupported span increases significantly, a threefold increase. When the bench cut method is used, $s=0.0001 p+1.2$; when CRD method is used, $s=0.0008 p+4.8$. So it can be seen that the unsupported span $s$ increases linearly as the action of $p$ increases. According to the actual project, we can choose whether to change the tunnel construction method to increase the unsupported span or to increase the unsupported span by increasing the support of the face (core soil).

\section{Analysis and Discussion}

It can be seen from (9) and (21) that the unsupported span of the tunnel is related to the cohesion of the surrounding rock $c$, the internal friction angle $\phi_{0}$, the weight $\gamma$, the thickness of the covering soil $H$, and the tunnel section sizes $d$ and $h$. In order to more clearly understand the main mechanical parameters of the soil mass and the relationship between the tunnel depth and the unsupported span, the parameters sensitivity analyses of the tunnel section sizes $d$ and $h$, buried depth $H$, cohesion $c$, and internal friction angle $\phi_{0}$ were carried out. When studying the relationship between the buried depth and the unsupported span, the tunnel depth is in the range of $h_{a}<H \leq(2-2.5) h_{a}$. When studying the relationship between cohesion, internal friction angle, and unsupported span, the values of $c$ and $\phi_{0}$ are in the soft rock range. 
TABLE 2: Stability of tunnel face and vault under different unsupported span.

\begin{tabular}{|c|c|c|c|c|}
\hline The unsupported span & $2.0 \mathrm{~m}$ & $3.0 \mathrm{~m}$ & $4.5 \mathrm{~m}$ & $5 \mathrm{~m}$ \\
\hline State description & $\begin{array}{l}\text { The tunnel face is stable, } \\
\text { with a small amount of } \\
\text { slump }\end{array}$ & $\begin{array}{l}\text { The arch crown collapsed and } \\
\text { the tunnel face began to break }\end{array}$ & $\begin{array}{l}\text { Both the arch crown } \\
\text { and the face failed }\end{array}$ & $\begin{array}{l}\text { The arch crown and the } \\
\text { face entirely are destroyed }\end{array}$ \\
\hline
\end{tabular}

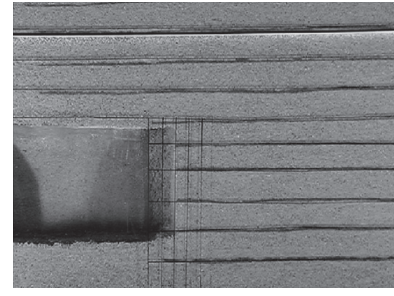

(a)

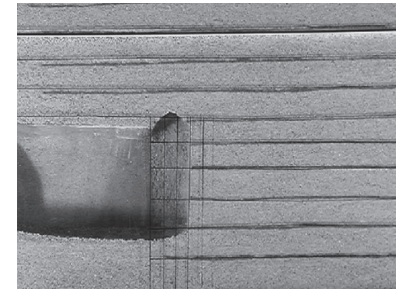

(b)

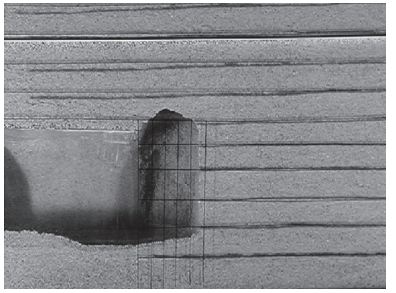

(c)

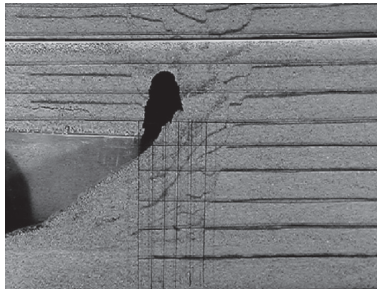

(d)

FIGURE 8: Model tests of the tunnel face and the unsupported span [19]. (a) The unsupported span is $2.0 \mathrm{~m}$. (b) The unsupported span is $3.0 \mathrm{~m}$. (c) The unsupported span is $4.5 \mathrm{~m}$. (d) The unsupported span is $5.0 \mathrm{~m}$.

TABLE 3: Calculation parameters.

\begin{tabular}{lccc}
\hline Weight $\gamma\left(\mathrm{kN} / \mathrm{m}^{3}\right)$ & Cohesion $c(\mathrm{kPa})$ & Fractional angel $\phi_{0}\left(^{\circ}\right)$ & Lateral pressure coefficient $K_{a}$ \\
\hline 20 & 50 & 22 & 0.45 \\
\hline
\end{tabular}

TABLE 4: Calculation of the unsupported span.

\begin{tabular}{|c|c|c|c|c|}
\hline Tunnel excavation method & Literature [15] & Literature [16] & This paper $(\mathrm{m})$ & On-site \\
\hline$\overline{C R D}$ & Unrestricted & $K<4.6$ & 4.8 & $3 \sim 5 \mathrm{~m}$ \\
\hline Bench cut method & $2 \mathrm{~m}$ & $K<2.9$ & 1.2 & $1 \sim 1.5 \mathrm{~m}$ \\
\hline
\end{tabular}

\subsection{The Relationship between Unsupported Span and Internal} Friction Angle. When $\gamma=20 \mathrm{kN} / \mathrm{m}^{3}, H=20 \mathrm{~m}, h=5 \mathrm{~m}$, and $d=10 \mathrm{~m}$, under the action of different cohesive forces, the unsupported span of the tunnel increases with the increase of the internal friction angle, as shown in Figure 9.

It can be seen from Figure 9 that, regardless of the cohesive force, the unsupported span of keeping the face stability increases with the increase of the internal friction angle; the unsupported span of keeping the arch crown stability increases with the increase of the internal friction angle when $\phi_{0}<27^{\circ}$; it decreases with the increase of the internal friction angle when $\phi_{0}>27^{\circ}$ but does not change as the cohesion force changes. When $c<60 \mathrm{kPa}$, it can be seen from the figure that the curve of the unsupported span of keeping face stability is below the curve of the unsupported span of keeping the arch crown stability. Therefore, when $c<60 \mathrm{kPa}$, the unsupported span of the tunnel is determined by the face stability. When $c \geq 110 \mathrm{kPa}$, the curve of the unsupported span of keeping the face stability is above the curve of the unsupported span of the arch crown stability. Therefore, when $c \geq 110 \mathrm{kPa}$, the unsupported span of the tunnel is determined by the arch crown stability; when $60 \mathrm{kPa}<c \leq 110 \mathrm{kPa}$, the curve of the unsupported span of keeping the face stability and the curve of the unsupported span of keeping the arch crown stability intersect each other. With the increase of cohesion, the curve of the unsupported

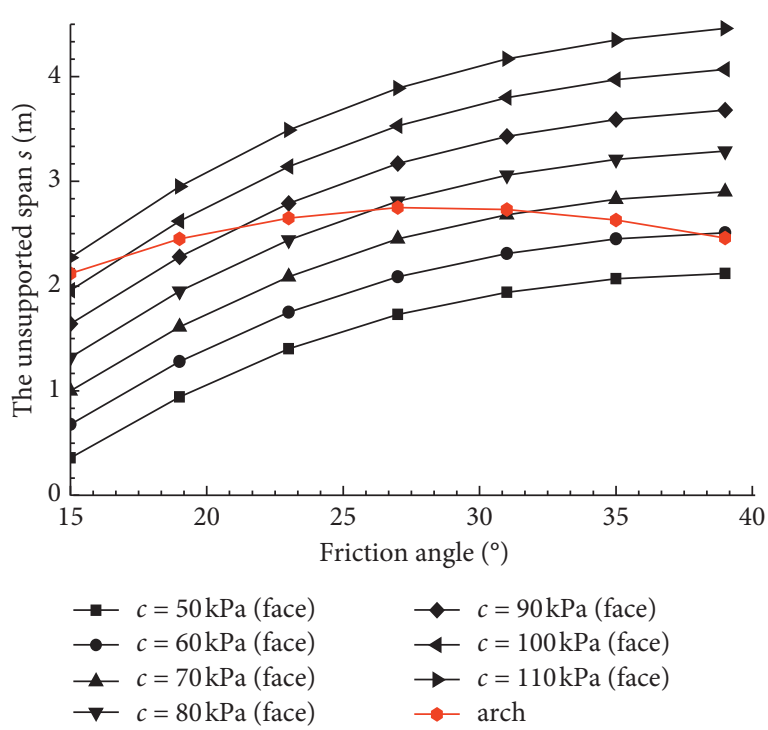

Figure 9: Curves of correlation between $s$ and $\phi_{0}$.

span of keeping the face stability rises until it exceeds the curve of the unsupported span of keeping the arch crown stability. Therefore, when $60 \mathrm{kPa}<c \leq 110 \mathrm{kPa}$, the unsupported span of the tunnel is determined by the internal friction angle. In summary, it can be proved that both 
determine the unsupported span of the tunnel; that is, the unsupported span of the tunnel $s=\min \{(6),(18)\}$.

When the relationship between the unsupported span and the internal friction angle is analyzed, the internal friction angle is between $15^{\circ}$ and $39^{\circ}$. However, for some sand and sand pebble stratum, the internal friction angle may be higher than this range. Therefore, a curve is added to further analyze the variation law of the unsupported span with the internal friction angle. When $\gamma=20 \mathrm{kN} / \mathrm{m}^{3}, H=20 \mathrm{~m}, h=5 \mathrm{~m}, d=10 \mathrm{~m}$, and $c=0 \mathrm{kPa}$, under the action of different cohesive forces, the change rule of the unsupported span of the face is shown in Figure 10.

It can be seen from Figure 10 that as the internal friction angle increases, the unsupported span of keeping the face and arch crown stability first increases and then decreases. Since the internal friction angle $\phi_{0}$ increases, the value also increases $\beta$, and the area of the fracture surface reduces so that the resultant force of the frictional force on the fracture surface continuously reduces. When the internal friction angle increases within a specific range, the resultant force of the friction force decreases, but the resultant force of the friction force is still higher than the gravity of the loose rock mass, so the unsupported span increases with the increase of the internal friction angle. When the internal friction angle exceeds a specific value, the continuously increased internal friction angle makes the resultant force of the frictional force fail to balance the gravity of the loose rock mass, and thus the unsupported span decreases as the internal friction angle increases.

4.2. The Relationship between Unsupported Span and Cohesion. When $\gamma=20 \mathrm{kN} / \mathrm{m}^{3}, H=20 \mathrm{~m}, h=5 \mathrm{~m}$, and $d=10 \mathrm{~m}$, under the action of different internal friction angles, the unsupported span of keeping the tunnel face stability increases linearly with the increase of cohesion; the unsupported span of keeping the arch crown stability increases first and decreases then with the increase of the cohesion. It is shown in Figure 11.

\subsection{The Relationship between Unsupported Span and Sectional} Dimension. When $\gamma=20 \mathrm{kN} / \mathrm{m}^{3}, H=20 \mathrm{~m}, \phi_{0}=27^{\circ}$, and $d=10 \mathrm{~m}$, under the action of different cohesive forces, the unsupported span of keeping the tunnel face stability decreases with the increase of the section width; the unsupported span of keeping the arch crown stability varied with variation of the section width, as shown in Figure 12(a). When $\gamma=20 \mathrm{kN} / \mathrm{m}^{3}, H=20 \mathrm{~m}, \phi_{0}=27^{\circ}$, and $h=7 \mathrm{~m}$, under the action of different cohesive forces, the unsupported span of keeping the tunnel face stability decreases with the increase of the section height; the unsupported span of keeping the arch crown stability decreases with the increase of the height of the section, as shown in Figure 12(b).

4.4. The Relationship between Unsupported Span and $H$. When $\gamma=20 \mathrm{kN} / \mathrm{m}^{3}, \phi_{0}=27^{\circ}, h=5 \mathrm{~m}$, and $d=10 \mathrm{~m}$, under the action of different cohesive forces, the unsupported span

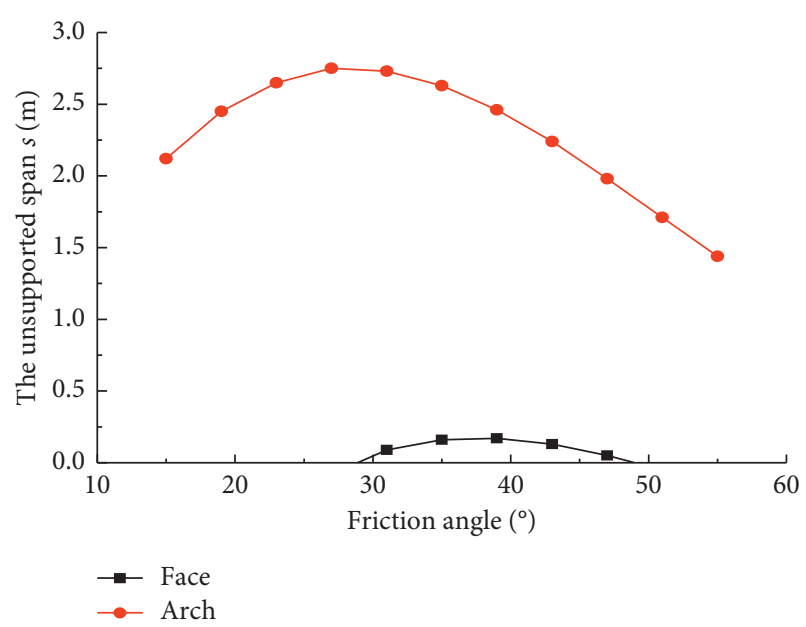

Figure 10: Curves of correlation between the unsupported span and internal friction angle.

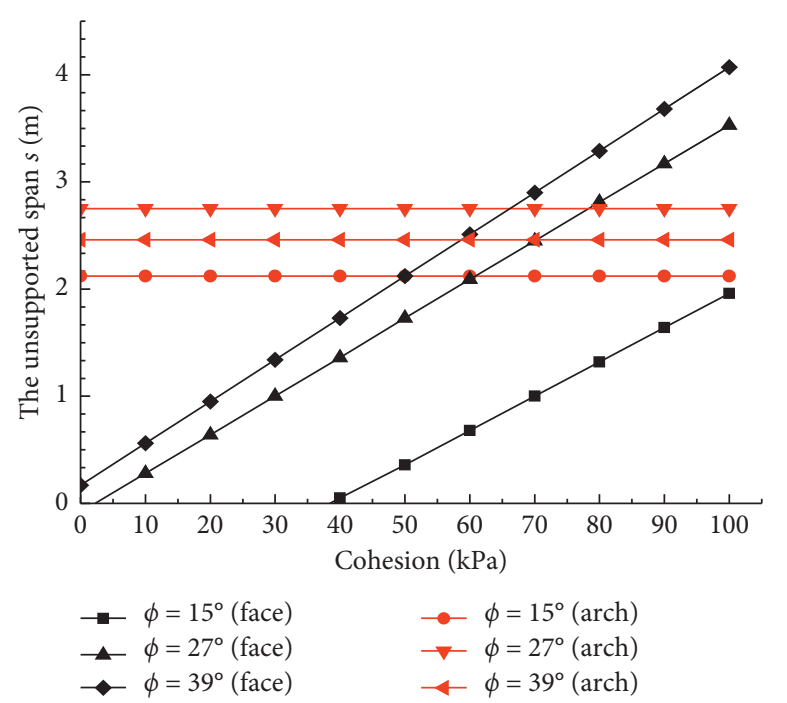

FIGURE 11: Curves of correlation between the unsupported span and cohesion.

of the tunnel increases with the increase of the tunnel depth; the unsupported span of keeping the arch crown stability decreases with the increase of the tunnel depth, as shown in Figure 13. When $c<60 \mathrm{kPa}$, the curve of the unsupported span of keeping the tunnel face stability is below the curve of the unsupported span of keeping the arch crown stability; when $c \geq 110 \mathrm{kPa}$, the curve of the unsupported span of keeping the face stability is above the curve of the unsupported span of the arch crown stability. When $60 \mathrm{kPa}<c \leq 110 \mathrm{kPa}$, the curve of the unsupported span of keeping the tunnel face stability and the curve of the unsupported span of keeping the arch crown stability intersect each other. As the cohesion increases, the curve of the unsupported span of keeping the tunnel face stability continues to rise until it exceeds the curve of the unsupported span of keeping the arch crown stability. Figure 13 proves again that both determine the unsupported span of the tunnel. 


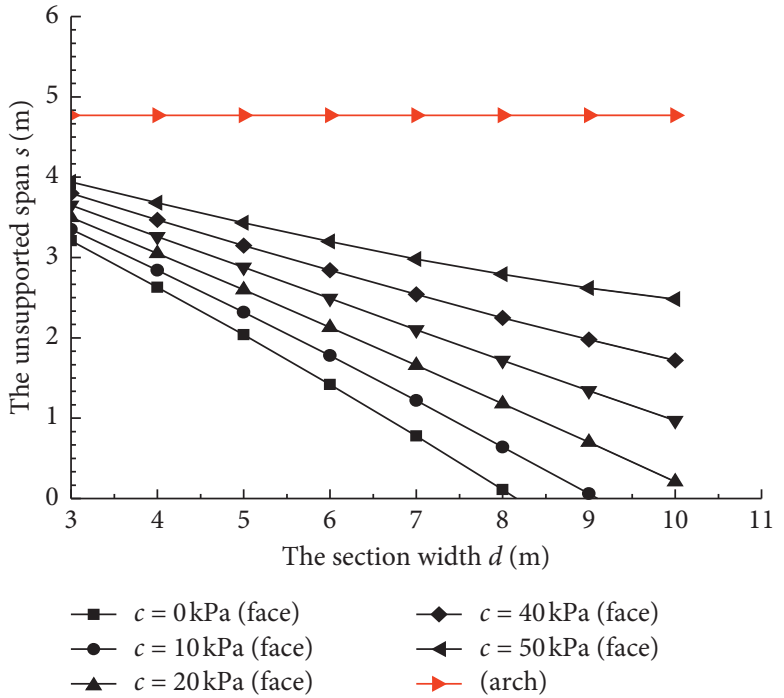

(a)

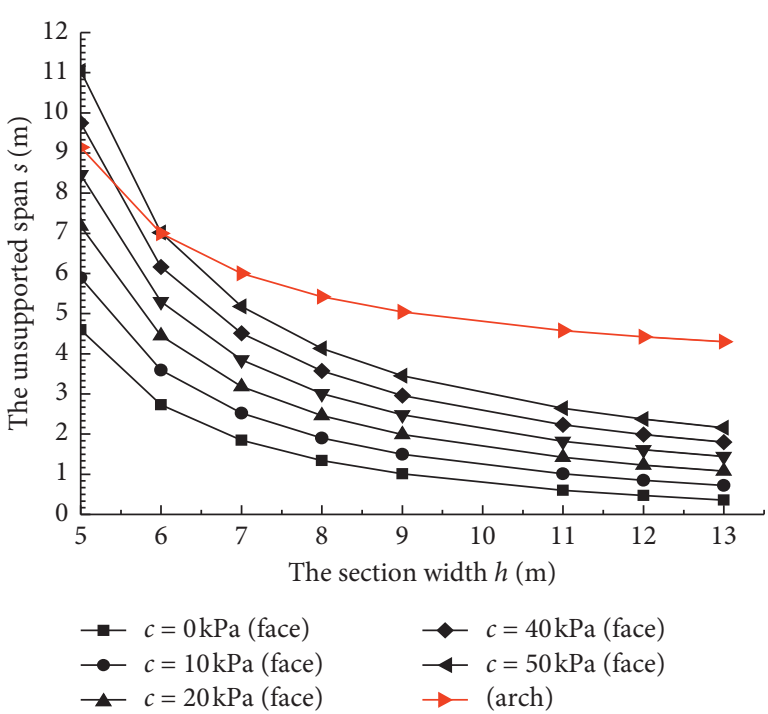

(b)

FIGURE 12: Curves of correlation between the unsupported span and sectional dimension. (a) The section width d. (b) The section height h.

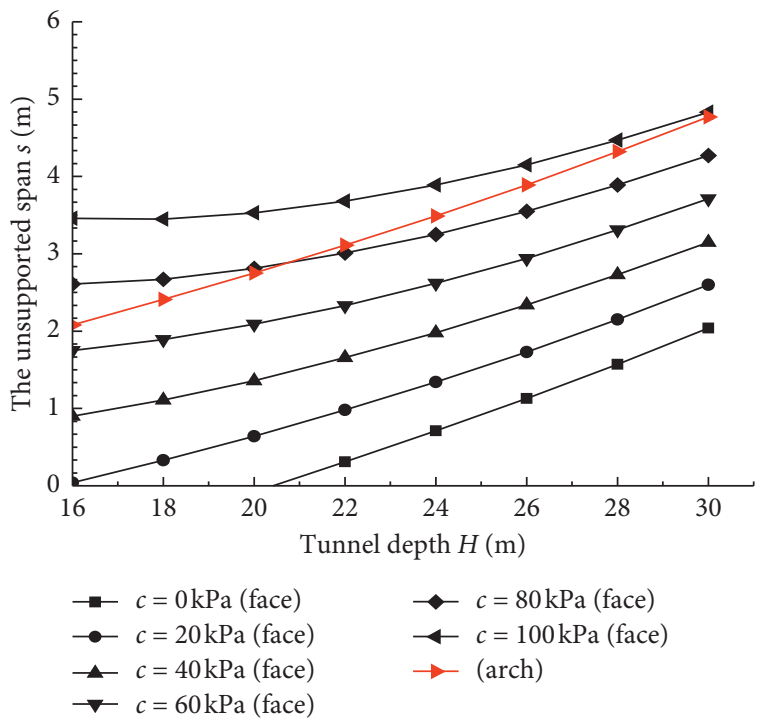

FIgURE 13: Curves of correlation between the unsupported span and depth of the tunnel.

In summary, the unsupported span of the tunnel is sensitive to the cohesion and internal friction angle of the rock-soil mass, especially the cohesion. Therefore, in practice, we should pay special attention to the cohesion and internal friction angle in calculating the unsupported span of the tunnel.

4.5. The Relationship between Unsupported Span and the Support Pressure. For the unsupported span of the tunnel face stability control, the unsupported span is relative to the stratum parameters and tunnel excavation dimensions [31]. In addition, the support pressure of the tunnel face has an essential influence on the unsupported

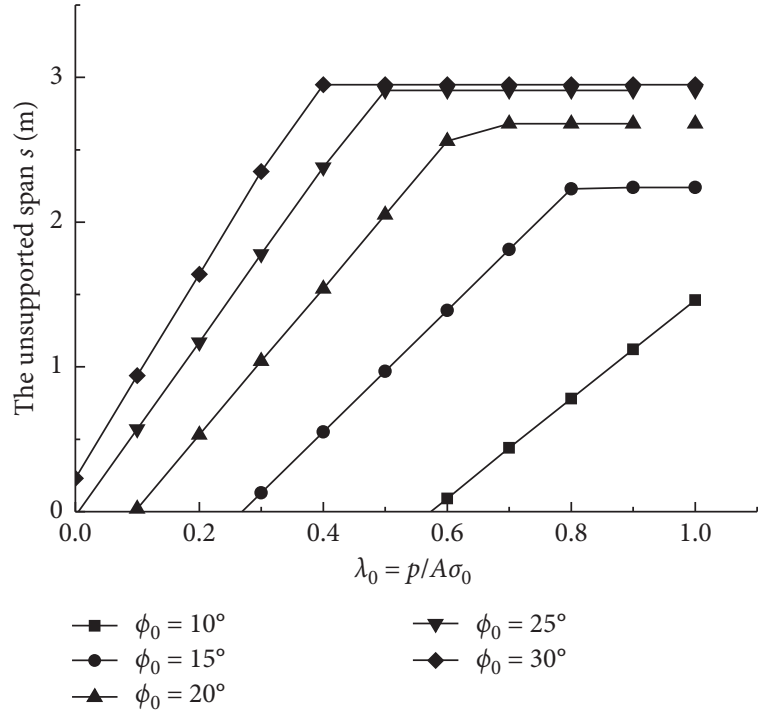

FIgURE 14: Curves of correlation between the unsupported span and limiting support ratio.

span. The support pressure on the excavation face is distributed in a trapezoid. For the convenience of explanation, the pressure value at the centre point of the excavation face is taken to represent the magnitude of the support pressure. At the same time, the support pressure ratio $\lambda_{0}$ is used to measure the support pressure of the excavation face:

$$
\lambda_{0}=\frac{p}{A \sigma_{0}}
$$

where $A$ is the area of the excavation face and $\sigma_{0}$ is the static earth pressure at the centre point of the excavation face.

When $\gamma=20 \mathrm{kN} / \mathrm{m}^{3}, H=20 \mathrm{~m}, h=5 \mathrm{~m}, d=8 \mathrm{~m}$, and $c=0$, under different support pressure ratios, the 
unsupported span of the tunnel changes with the internal friction angle, as shown in Figure 14. It can be seen from Figure 14 that the curve of the unsupported span changing with the support pressure ratio is composed of two parts. The oblique section is the unsupported span of the tunnel face of the stability control, and the straight section is the unsupported span of the arch crown of stability control. As the support pressure ratio increases, the unsupported span of keeping the tunnel face stability increases linearly, and the curve changes to that of unsupported span controlled by the arch crown stability when it increases to a certain extent. As the internal friction angle increases, the slope of the oblique line segment also increases, indicating that as the internal friction angle increases, the contribution of the support pressure to the excavation footage is increasingly higher. It is in line with the actual situation of the project, which shows that the formula pushed in this paper has certain rationality.

\section{Conclusions}

A theoretical method of the unsupported span for the shallow tunnel in soft stratum was proposed in this study. The rationality of the formula was verified by a centrifugal test and an engineering case. The conclusions obtained are as follows:

(1) It was concluded that the unsupported span of the tunnel is not determined by the arch crown stability or the tunnel face stability alone, but by both.

(2) Based on the calculation model of the surrounding rock pressure of shallow buried tunnel, this paper analyzed the stability of the tunnel face and the vault and then obtained the calculation formula of the unsupported span of the shallow buried tunnel in soft rock stratum. The analysis and discussion show that the unsupported span is sensitive to the cohesion and internal friction angle of the rock-soil mass, especially the cohesion. In the actual project, we should pay special attention to the values of cohesion and internal friction angle in calculating the unsupported span of the tunnel.

(3) The unsupported span $s$ of the shallow buried tunnel in the soft rock stratum is a linear function of the action of $p$. According to the calculation results of the engineering example, the calculation formula proposed is in line with the engineering practice. The action of $p$ has a more significant contribution to the increase of the unsupported span by the CRD method, and the unsupported span $s$ increases linearly with the increase of the action of $p$.

\section{Data Availability}

The data used to support the findings of this study are available from the corresponding author upon request.

\section{Conflicts of Interest}

The authors declare that they have no conflicts of interest.

\section{Acknowledgments}

The present work was subsidised and supported by the National Natural Science Foundation of China (No. 51578447), the Science and Technology Development Program of Shaanxi Provincial Department of Housing and Urban-Rural Construction (No. 2019-K39), the China Postdoctoral Science Foundation (No. 2018M643809XB), and the Natural Science Basic Research Program of Shaanxi (No. 2019JQ-762). The authors gratefully acknowledge the financial supports.

\section{References}

[1] X. X. Tian, Z. P. Song, and J. B. Wang, "Study on the propagation law of tunnel blasting vibration in stratum and blasting vibration reduction technology," Soil Dynamics and Earthquake Engineering, vol. 126, Article ID 105813, 2019.

[2] Z. Chu, Z. Wu, Q. Liu, and B. Liu, "Analytical solutions for deep-buried lined tunnels considering longitudinal discontinuous excavation in rheological rock mass," Journal of Engineering. Mechanics, vol. 146, no. 6, Article ID 04020047, 2020.

[3] K. Wu, Z. Shao, S. Qin, and B. Li, "Determination of deformation mechanism and countermeasures in silty clay tunnel," Journal of Performance of Constructed Facilities, vol. 34, no. 1, Article ID 04019095, 2020.

[4] Z. P. Song, Y. Cheng, X. X. Tian, J. Wang, and T. Yang, "Mechanical properties of limestone from Maixi tunnel under hydro-mechanical coupling," Arabian Journal of Geosciences, vol. 13, no. 11, pp. 1-11, 2020.

[5] X. Tian, Z. Song, B. Wang, and G. Zhou, "A theoretical calculation method of influence radius of settlement based on the slices method in tunnel construction," Mathematical Problems in Engineering, vol. 2020, no. 2, 9 pages, Article ID 5804823, 2020.

[6] K. Wu and Z. Shao, "Visco-elastic analysis on the effect of flexible layer on mechanical behavior of tunnels," International Journal of Applied Mechanics, vol. 11, no. 3, Article ID 1950027, 2019.

[7] X. L. Huang, Z. G. Zhou, and X. L. Yang, "Roof failure of shallow tunnel based on simplified stochastic medium theory," Geomechanics and Engineering, vol. 14, no. 6, pp. 571-580, 2018.

[8] P. Guo, H. Li, G. Zhang, and Y. Zhang, "Exploring critical variables that affect the policy risk level of industrial heritage projects in Chinaffect the policy risk level of industrial heritage projects in China," Sustainability, vol. 11, no. 23, p. 6848, 2019.

[9] N. Khezri, H. Mohamad, and B. Fatahi, "Stability assessment of tunnel face in a layered soil using upper bound theorem of limit analysis," Geomechanics and Engineering, vol. 11, no. 4, pp. 471-492, 2016.

[10] P. Guo, W. Tian, H. M. Li, G. Zhang, and J. Li, "Global characteristics and trends of research on construction dust: based on bibliometric and visualized analysis," Environmental Science and Pollution Research, vol. 27, 2020.

[11] I. Ocak, "A new approach for estimating the transverse surface settlement curve for twin tunnels in shallow and soft soils," Environmental Earth Sciences, vol. 72, no. 7, pp. 2357-2367, 2014.

[12] Y. W. Zhang, Z. P. Song, and X. L. Weng, "A constitutive model for loess considering the characteristics of structurality 
and anisotropy," Soil Mechanics and Foundation Engineering, vol. 57, 2020, in Press.

[13] J. L. Qiu, Y. Q. Lu, J. X. Lai, Y. Zhang, T. Yang, and K. Wang, "Experimental study on the effect of water gushing on loess metro tunnel," Environmental Earth Sciences, vol. 79, no. 11, pp. 1-12, 2020.

[14] Z. P. Song, X. X. Tian, Q. Liu, Y. Zhang, H. Li, and G. Zhou, "Numerical analysis and application of the construction method for small interval tunnel in the turn line of metro," Science Progress, vol. 103, no. 3, Article ID 003685042093206, 2020.

[15] Z. D. Wang and X. N. Gong, "Calculation method of digging length in underpass with underground excavation under shallow cover," Rock and Soil Mechanics, vol. 31, no. 8, pp. 2637-2641, 2010, in Chinese.

[16] X. H. Shi, Y. Q. Dai, and J. Q. Guo, "Calculation and analysis on excavation cycle length of shallow portal sections of tunnels located in lalus deposits:case study on Zhaojiawu tunnel on Ma-hao highway in Yunnan, China," Tunnel Construction, vol. 35, no. 8, pp. 787-791, 2015, in Chinese.

[17] X. Zhang, M. N. Wang, J. W. Li, Z. L. Wang, J. J. Tong, and D. G. Liu, "Safety factor analysis of a tunnel face with an unsupported span in cohesive-frictional soils," Computers and Geotechnics, vol. 117, Article ID 103221, 2020.

[18] Q. Liang, X. L. Yang, and X. Chen, "Limit equilibrium analysis of round length in tunnel excavation," Huanan Ligong Daxue Xuebao/Journal of South China University of Technology (Natural Science), vol. 45, no. 5, pp. 113-119, 2017.

[19] Y.-Z. Lee and W. Schubert, "Determination of the round length for tunnel excavation in weak rock," Tunnelling and Underground Space Technology, vol. 23, no. 3, pp. 221-231, 2008.

[20] D. Kolymbas, Tunnelling and Tunnel Mechanics a Rational Approach to Tunnelling, Springer Ebooks, New York, NY, USA, 2005.

[21] P. Chambon and J. F. Corté, "Shallow tunnels in cohesionless soil: stability of tunnel face," Journal of Geotechnical Engineering, vol. 120, no. 7, pp. 1148-1165, 1994.

[22] H. Kamata and H. Mashimo, "Centrifuge model test of tunnel face reinforcement by bolting," Tunnelling and Underground Space Technology, vol. 18, no. 2-3, pp. 205-212, 2003.

[23] A. Kirsch, "Experimental investigation of the face stability of shallow tunnels in sand," Acta Geotechnica, vol. 5, no. 1, pp. 43-62, 2010.

[24] M. Ahmed and M. Iskander, "Evaluation of tunnel face stability by transparent soil models," Tunnelling and Underground Space Technology, vol. 27, no. 1, pp. 101-110, 2012.

[25] X. L. Yang and F. Huang, "Three-dimensional failure mechanism of a rectangular cavity in a hoek-brown rock medium," International Journal of Rock Mechanics and Mining Sciences, vol. 61, no. 10, pp. 189-195, 2013.

[26] M. Fraldi and F. Guarracino, "Evaluation of impending collapse in circular tunnels by analytical and numerical approaches," Tunnelling and Underground Space Technology, vol. 26, no. 4, pp. 507-516, 2011.

[27] G. Mollon, D. Dias, and A.-H. Soubra, "Rotational failure mechanisms for the face stability analysis of tunnels driven by a pressurized shield," International Journal for Numerical and Analytical Methods in Geomechanics, vol. 35, no. 12, pp. 1363-1388, 2011.

[28] R. J. Mair and R. N. Taylor, "Bored tunnelling in the urban environment," in Proceedings of the 14th International Conference on Soil Mechanics and Foundation Engineering, Hamburg, Germany, September 1997.
[29] A. R. Selby, "Surface movements caused by tunnelling in twolayer soil," Geological Society, London, Engineering Geology Special Publications, vol. 5, no. 1, pp. 71-77, 1988.

[30] J. J. Xie, "Stratigraphic pressure of shallow tunnel," China Civil Engineering Journal, vol. 10, no. 6, pp. 58-70, 1964, in Chinese.

[31] W. Broere, "On the face support of microtunnelling TBMs," Tunnelling and Underground Space Technology, vol. 46, pp. 12-17, 2015. 